\title{
Using an RNA aptamer probe for flow cytometry detection of CD30-expressing lymphoma cells
}

\author{
Peng Zhang ${ }^{1,3}$, Nianxi Zhao ${ }^{1}$, Zihua Zeng ${ }^{1}$, Yongdong Feng ${ }^{1,3}$, Ching-Hsuan Tung ${ }^{2}$, Chung-Che Chang $^{1}$ and \\ Youli Zu $\mathrm{u}^{1, *}$
}

Aptamers are small molecular ligands composed of short oligonucleotides that bind targets with high affinity. In contrast to antibodies, as synthetic oligonucleotides, aptamers have lower production costs and elicit no antigenic reactions. Therefore, aptamers are potential agents for disease diagnosis and treatment. In this study, we validate a fluorescently labeled RNA aptamer, which has been reported to bind specifically to mouse CD30 proteins in solution, for human CD30 protein recognition on intact cells. The aptamer probe was tested with cultured anaplastic large cell lymphoma and Hodgkin's lymphoma cells that express high levels of CD30. Flow cytometry and fluorescence microscopy showed specific and sensitive binding of the aptamer probe to CD30-expressing lymphoma cells at low concentrations ( $0.3 \mathrm{nM})$. Studies performed on multiple cell lines and nuclear cells from healthy donors confirmed that the CD30 aptamer and anti-CD30 antibody, the standard clinical probe, recognized the same set of cells. The potential application of multicolor flow cytometry analysis using the CD30 aptamer probe and antibodies was also shown. In conclusion, the developed CD30 aptamer probe could act as a replacement and/or a supplement for antibodies in the diagnosis of the CD30-expressing lymphomas.

Laboratory Investigation (2009) 89, 1423-1432; doi:10.1038/labinvest.2009.113; published online 12 October 2009

KEYWORDS: aptamer probe; CD30 expression; cell binding; flow cytometry analysis; lymphoma diagnosis; RNA oligonucleotide

In the past two decades, a new class of small molecule ligands composed of short oligonucleotides (usually 20-50 bases), known as 'aptamers', has been widely investigated. ${ }^{1-7}$ Use of the systematic evolution of ligands by EXponential amplification technique has led to a variety of RNA or singlestranded DNA aptamers that have high binding affinity to their target molecules at pico- to nanomolar levels. ${ }^{7,8}$ Aptamers can virtually bind to all types of targets, including ions, toxins, drugs, low-molecular-weight ligands, peptides, proteins, and cells. A recent study that used the extracellular domain of the receptor activator of nuclear factor- $\kappa \mathrm{B}$ (RANK) as a target identified an RNA aptamer that specifically binds to the tumor necrosis factor (TNF) receptor family proteins. ${ }^{9}$ Interestingly, among its binding targets, this aptamer binds to mouse CD30 proteins with the highest affinity, more than 1000 times higher than other tested proteins among the TNF receptor family members in solution.
CD30 antigen is a transmembrane receptor that is normally expressed by activated $\mathrm{T}$ cells. ${ }^{10}$ Abnormal expression of CD30 has been identified in tumor cells of anaplastic large cell lymphoma (ALCL) and Hodgkin's lymphoma. ${ }^{11,12}$ Detection of CD30 expression on tumor cells is a requirement for diagnosis of these lymphomas and for differentiating them from other types of tumors. ${ }^{13}$ To date, the anti-CD30 antibody is the only probe that has been validated to detect cellular CD30 expression in clinical settings. ${ }^{14}$ It is known that the binding of cell surface CD30 to its ligands could trigger complicated cellular responses and might be involved in the pathogenesis of the CD30-expressing neoplasms. ${ }^{15-17}$ Thus, cellular CD30 receptor targeting represents a new therapeutic strategy for both diagnosing and treating ALCL. ${ }^{18-22}$ In contrast to protein antibodies, aptamers are small, synthetic oligonucleotides, and therefore they are not antigenic in vivo and show better tissue/cell penetration. ${ }^{7,23}$ To validate the potential usage of aptamers in lymphoma

\footnotetext{
'Department of Pathology, The Methodist Hospital and The Methodist Hospital Research Institute, Houston, TX, USA and ${ }^{2}$ Department of Radiology, The Methodist Hospital and The Methodist Hospital Research Institute, Houston, TX, USA

*Correspondence: Dr Y Zu, MD, PhD, Department of Pathology, The Methodist Hospital, 6565 Fannin Street, Houston, TX 77030, USA.

E-mail: yzu@tmhs.org

${ }^{3}$ Current address: Department of General Surgery, Tongji Hospital, Huazhong University of Science and Technology, China.

Received 29 May 2009; revised 10 August 2009; accepted 25 August 2009
} 
diagnosis, we tested the previously identified RNA aptamer as a specific probe to detect the human CD30-expressing lymphoma cells. Furthermore, the usage of multicolor immunophenotyping was shown by combining the fluorescently labeled aptamer probe and antibodies.

\section{MATERIALS AND METHODS}

\section{Cell Culture, Cell Preparation, and Reagents}

In this study, the human systemic ALCL cell lines Karpas 299 and SUDHL-1 were used in collaboration with Dr Mark Raffeld at the National Cancer Institute/National Institutes of Health. The human acute T-cell leukemia cell line Jurkat, human myeloid leukemia cell line K562, and human myeloma cell line RPMI8226 were purchased from the American Type Culture Collection (ATCC, Manassas, VA, USA). Human Hodgkin's lymphoma cell lines, L540, HDLM2, L428, and $\mathrm{KMH} 2$ were used in collaboration with Dr Barbara Savoldo at the Baylor College of Medicine (Houston, TX, USA). Cells were routinely cultured in RPMI-1640 medium (Mediatech, Manassas, VA, USA) supplemented with $10 \%$ fetal bovine serum (FBS; Invitrogen, Carlsbad, CA, USA), 100 units $/ \mathrm{ml}$ penicillin, and $100 \mu \mathrm{g} / \mathrm{ml}$ streptomycin (Atlanta Biologicals, Lawrenceville, GA, USA) at $37^{\circ} \mathrm{C}$ in a $5 \% \mathrm{CO}_{2}$ atmosphere.

For preclinical evaluation, nuclear cells were prepared from bone marrow aspirates from healthy donors under an Institutional Review Board approved protocol $(n=10)$. In brief, $1 \mathrm{ml}$ anticoagulant-treated bone marrow specimens were centrifuged at $500 \mathrm{~g}$ for $10 \mathrm{~min}$, and nuclear cells within the Buffy coat between the serum and cell pellets were collected. Contaminated red blood cells (RBCs) were lysed by $6 \mathrm{ml}$ of RBC lysis buffer (Cell Signaling Technology, Inc., Danvers, MA, USA) at room temperature for $10 \mathrm{~min}$. Unlysed nuclear cells were then washed with $2 \mathrm{ml}$ phosphatebuffered saline (PBS, pH 7.4) and resuspended in cold PBS. In addition, to prepare fresh lymphoma cells, the patients' lymph nodes involved by follicular lymphoma $(n=5)$ were digested by vigorous cutting and repeated pipetting to generate a cell suspension in cold PBS. The cells were then filtered through a $100 \mu \mathrm{m}$ cup filcon (BD Medimachine, San Jose, CA, USA) to obtain a single-cell suspension.

\section{Preparation of RNA Aptamer Probe Specific for CD30}

According to the reported aptamer sequences, ${ }^{9}$ a 39 -mer nucleotide RNA aptamer was synthesized with one minor modification: $5^{\prime}$-gauUCGUAUGGGUGGGAUCGGGAAGGG CUACGAACAccg-3' (Bio-Synthesis, Inc., Lewisville, TX, USA). The synthesized aptamer probe contains the most essential functional motif and has the highest affinity to mouse CD30 molecules in solution. ${ }^{9}$ To enhance its resistance to nucleases, the first three nucleotides on each end of the aptamer were synthesized with 2'-O-methyl modification (indicated by lower case letters). The predicted 3D structure of the aptamer is shown in Figure 1a. For tracking purposes, the synthesized aptamers were labeled with the fluorochrome cyanine dye, Cy5, at the $5^{\prime}$ end. The aptamers were purified by high performance liquid chromatography and stored at $-20^{\circ} \mathrm{C}$ until use. To rule out nonspecific cell binding, a control 39-mer RNA probe was synthesized with a random sequence and also labeled with the Cy5 reporter: $5^{\prime}$-gau- random $\mathrm{rN}(n=33)$-ccg- $3^{\prime}$ (Figure 1a).

\section{Cell Binding Assay and Flow Cytometry Analysis}

To determine the optimal cell binding conditions for the synthesized CD30 aptamer probes, Karpas 299 cells that express CD30 and Jurkat cells that do not express CD30 were used. Cells $\left(5 \times 10^{5} /\right.$ assay $)$ were incubated with the CD30 aptamer probes $(0.3 \mathrm{nM})$ in $100 \mu$ l of cell binding buffer (see below) at room temperature for $20 \mathrm{~min}$ and then washed once with binding buffer without bovine serum albumin (BSA). The cells were analyzed by an LSRII flow cytometer (BD Biosciences, San Jose, CA, USA) with FlowJo (v7.0) software. To validate the optimal cell binding conditions, different concentrations of $\mathrm{CaCl}_{2}, \mathrm{MgCl}_{2}$, and BSA (SigmaAldrich, St. Louis, MO, USA) in PBS were tested as indicated in Figure 2. After confirmation, the optimized binding buffer (PBS, pH 7.4) containing $1 \mathrm{mM} \mathrm{MgCl}_{2}$ and $0.1 \%$ BSA was used throughout the remaining experiments. To determine the optimal concentration of the $\mathrm{CD} 30$ aptamer probe, a dosing experiment was performed using different concentrations of aptamer probes $(0-3 \mathrm{nM})$ as described in Figure 2. A standard concentration for cell binding assays of the $0.3 \mathrm{nM}$ CD30 aptamer was selected for all additional experiments.

To validate the binding specificity of the CD30 aptamer, various cultured lymphoma and leukemia cell lines were used as listed in Table 1. Cells were incubated with the Cy5-labeled $\mathrm{CD} 30$ aptamer, as described above, or the fluorescein isothiocyanate (FITC)-labeled anti-CD30 antibody (BD Biosciences), a standard probe for examining CD30-expressing cells in clinical laboratories. For cell staining, the $0.3 \mathrm{nM}$ CD30 aptamer or 1:20 diluted anti-CD30 antibody was added to $5 \times 10^{5}$ cells in $100 \mu \mathrm{l}$ binding buffer and incubated at room temperature for $20 \mathrm{~min}$. After washing with binding buffer without BSA, probe or antibody binding was detected by flow cytometry analysis. For further validation, nuclear cells from healthy donor bone marrow $(n=10)$, which included granulocytes, monocytes, lymphocytes, and nuclear RBCs, and fresh lymphoma cells from patients' lymph nodes involved by follicular lymphoma $(n=5)$ were stained with the CD30 aptamer or anti-CD30 antibody.

To examine whether the CD30 aptamer and CD30 antibody compete for the same binding site on the CD30 protein, cultured Karpas 299 cells $\left(5 \times 10^{5}\right)$ were co-stained with the Cy5-labeled CD30 aptamer $(0.3 \mathrm{nM})$ and FITC-labeled CD30 antibody (1:20 dilution) for $20 \mathrm{~min}$. Cell staining by each probe was evaluated by flow cytometry analysis.

To determine whether the CD30 aptamer probes could be combined with other antibodies to produce multicolor cell staining, phycoerythrin (PE)-labeled anti-CD25 antibody, FITC-labeled anti-CD30 antibody, and AmCyan-labeled anti- 
a

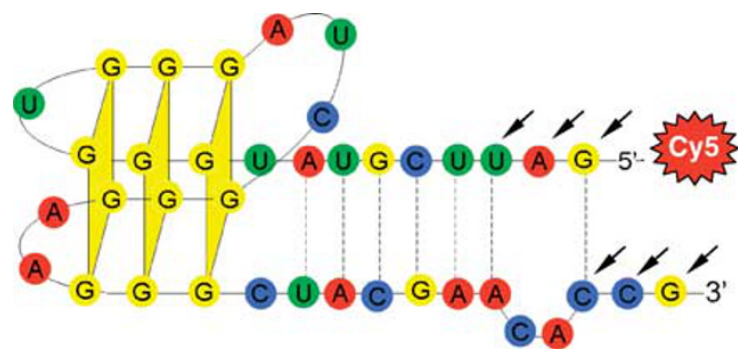

CD30 aptamer: 5'-gauUCGUAUGGGUGGGAUCGGGAAGGGCUACGAACAccg-3'

Control sequence: 5'-gauNNNNNNNNNNNNNNNNNNNNNNNNNNNNNNNNNccg-3'
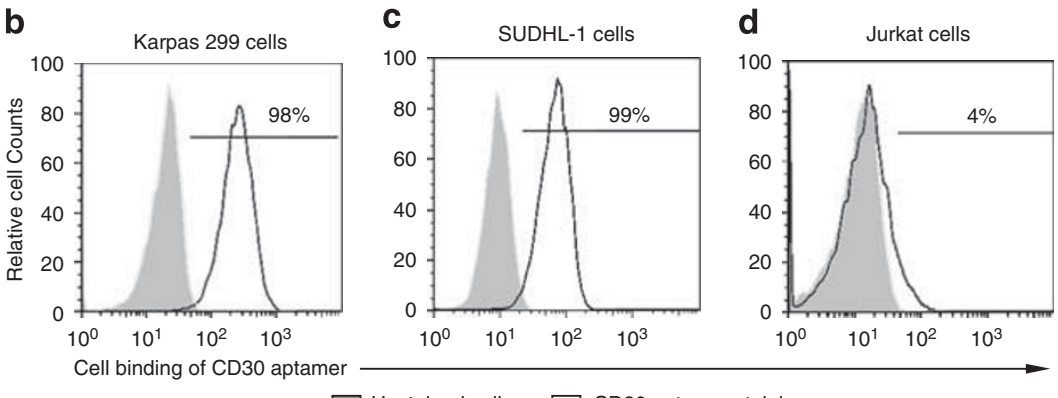

$\square$ Unstained cells $\square$ CD30 aptamer staining

Figure 1 Specific binding of the synthesized CD30 aptamer to human lymphoma cells. (a) The synthesized CD30 aptamer sequence and its predicted structure. A 39-mer RNA aptamer was synthesized according to the published sequence (9) and labeled with fluorochrome Cy5 at its $5^{\prime}$-end. To improve its nuclease resistance, the first three nucleotides on each end were synthesized with 2'-O-methyl modification (arrows). To rule out nonspecific cell binding, a control 39-mer RNA probe was synthesized with a random sequence and also labeled with the Cy5 reporter as illustrated. (b-d) Cell binding was assessed by flow cytometry analysis. Cultured human ALCL cells (Karpas 299 and SUDHL-1, which express a high level of CD30 proteins) and Jurkat cells that do not express CD30 were incubated with the $0.3 \mathrm{nM} \mathrm{CD30}$ aptamer, and the resultant aptamer-cell binding was detected by flow cytometry analysis. Gray peaks represent unstained cells, and open peaks represent cells that were stained with the CD30 aptamer.

CD45 antibody from BD Biosciences were tested. Cultured Karpas 299 cells, SUDHL-1 cells, and Jurkat cells $\left(5 \times 10^{5}\right)$ were stained with mixed probes, including the $0.3 \mathrm{nM}$ Cy5labeled aptamer, 1:20 anti-CD25 antibody, 1:20 anti-CD30 antibody, and 1:20 anti-CD45 antibody at room temperature for $20 \mathrm{~min}$. Cells were washed once, and binding of the aptamer and antibodies to cells was detected by an LSRII flow cytometer with four-color channels.

To detect ALCL cells in a heterogeneous cell specimen, fresh nucleated cells $\left(5 \times 10^{5}\right)$ from the bone marrows of healthy donors were prepared by lysing the RBCs using RBC lysis buffer as described above. A small amount of cultured Karpas 299 cells ( $\sim 0.5-13 \%$ of total cellularity) were added into the marrow cell specimens and the mixed cells were incubated simultaneously with the Cy5-labeled CD30 aptamer, AmCyan-labeled anti-CD45 antibody, and FITC-labeled anti-CD30 antibody for $20 \mathrm{~min}$ as described above. Stained cells were washed once and flow cytometry analysis was performed using an LSRII flow cytometer with three-color channels. The individual cellular populations in the cell mixture were separated and gated according to their distribution on the side scatter (SSC) vs CD45 panels following standard protocol for clinical flow cytometry (Figure 7a). Cellular CD30 expression in each gated cell population was examined using both the CD30 aptamer probe and anti-
CD30 antibody, and the numbers of detected CD30-positive Karpas 299 cells were compared.

\section{Cell Staining Analysis of CD30 Aptamer Probes by Fluorescence Microscopy}

Specific CD30 binding of aptamer was further examined using fluorescence microscopy. In this experiment, CD30negative Jurkat cells $\left(1 \times 10^{6}\right)$ were pre-labeled with $25 \mathrm{nM}$ of carboxyfluorescein diacetate succinimidyl ester (CFSE, Invitrogen, Eugene, OR, USA) in $1 \mathrm{ml}$ of PBS in the dark at $37^{\circ} \mathrm{C}$ for $15 \mathrm{~min} .^{24}$ The cells were then washed with PBS once and recultured in $4 \mathrm{ml}$ fresh RPMI 1640 medium containing $10 \%$ FBS for 30 min before use. A cell mixture was prepared by mixing the CFSE-labeled Jurkat cells $\left(5 \times 10^{5}\right)$ with an equal number of fresh Karpas 299 cells in binding buffer. Cy5-labeled CD30 aptamer probe $(0.3 \mathrm{nM})$ was added to the cell mixture and incubated at room temperature for $20 \mathrm{~min}$. After washing, slides of cell smears were prepared and examined under a fluorescent microscope.

\section{RESULTS \\ RNA Aptamer Specifically Binds to Cultured Human CD30-Expressing Lymphoma Cells}

To test whether the reported RNA aptamer recognizes proteins of the mouse TNF receptor family and is able to bind to 
a

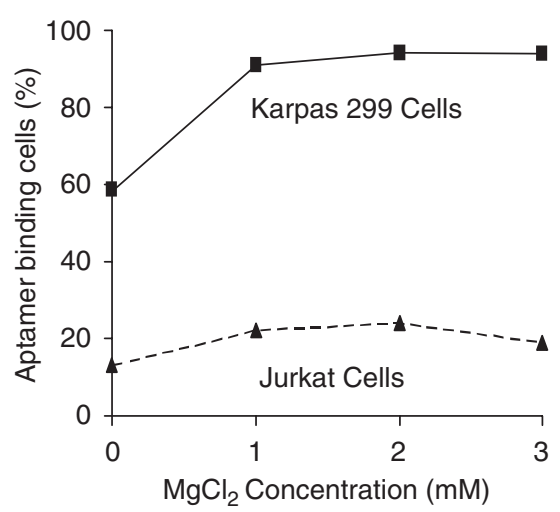

d

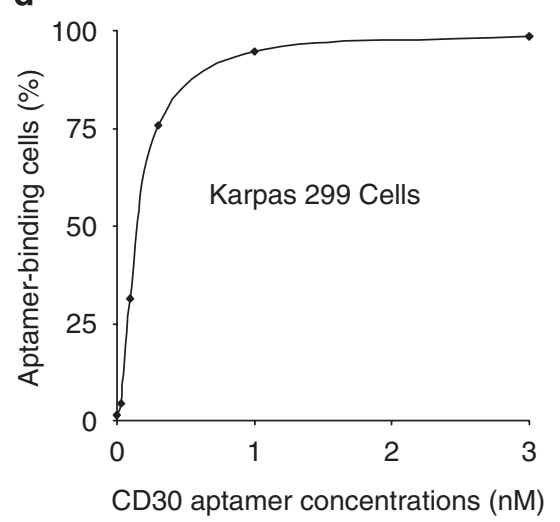

b

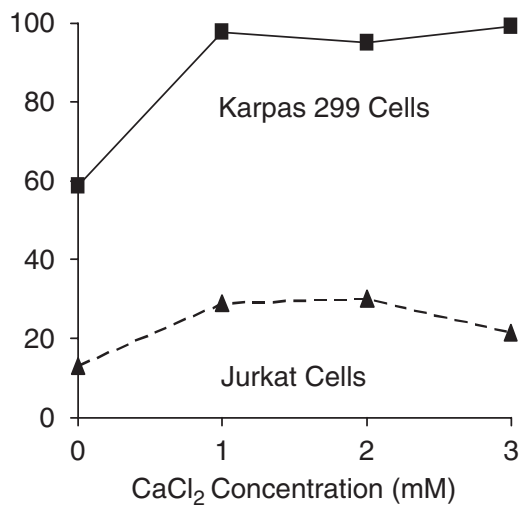

e

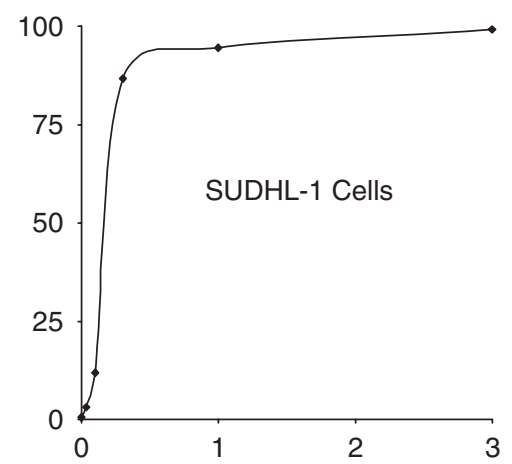

C

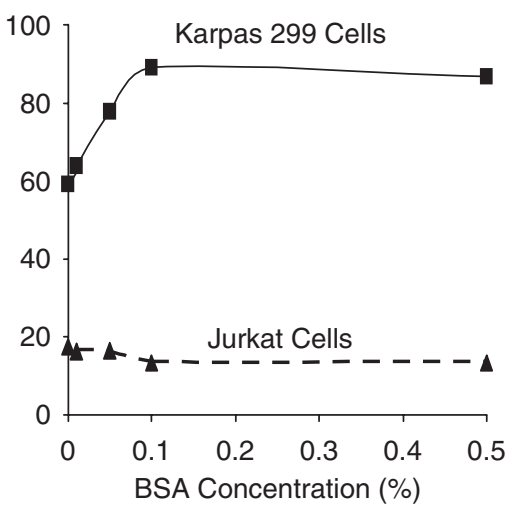

f

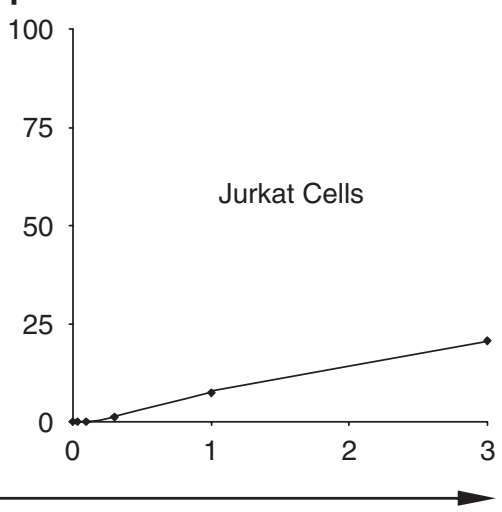

Figure 2 Optimal cell binding conditions for the CD30 aptamer. (a) Effect of $\mathrm{MgCl}_{2}$ on cell binding. Karpas 299 cells were incubated with the $\mathrm{CD} 30$ aptamer in PBS ( $\mathrm{pH}$ 7.4) with varying concentrations of $\mathrm{MgCl}_{2}$ ranging from 0 to $3 \mathrm{mM}$. Resultant aptamer-cell binding was detected by flow cytometry analysis. (b) Effect of $\mathrm{CaCl}_{2}$. The cell binding assay was repeated with varying concentrations of $\mathrm{CaCl}_{2}$ ranging from 0 to $3 \mathrm{mM}$. (c) Effect of $\mathrm{BSA}$. The cell binding assay was repeated with varying concentrations of BSA ranging from 0 to $0.5 \%$ (wt/wt). (d-f) Dosage study of the CD30 aptamer. Cultured Karpas 299 (d), SUDHL1 (e), and Jurkat (f) cells were incubated with increasing concentrations of CD30 aptamer from 0 to $3 \mathrm{nM}$ as indicated. The resultant aptamer-cell binding (\%) was detected by flow cytometry analysis.

human CD30 on intact cells, a 39-mer RNA aptamer was synthesized according to the published sequence ${ }^{9}$ and labeled with fluorochrome Cy5 (Figure 1a). The first three nucleotides on each end of the aptamer were replaced with $2^{\prime}$-O-methyl nucleotides for better nuclease resistance (indicated by arrows). To assess cell binding, Karpas 299 and SUDHL-1 cells, which are human ALCL cell lines that characteristically express high levels of CD30 on their surfaces, were tested. Jurkat cell, a human T-cell leukemia cell line that does not express CD30, was used as a negative control. Cells were incubated with the CD30 aptamer probes $(0.3 \mathrm{nM})$ in binding buffer for $20 \mathrm{~min}$ at room temperature, and resultant cell binding of the CD30 aptamer probe was quantitated by flow cytometry analysis. As shown in Figures $1 \mathrm{~b}-\mathrm{d}$, the CD30 aptamer probes only bound to Karpas 299 and SUDHL-1 cells, but not to Jurkat cells. More than $98 \%$ of the Karpas 299 and SUDHL-1 cells were labeled with the probes.

To optimize binding conditions for the CD30 aptamer, PBS buffer ( $\mathrm{pH}$ 7.4) with varying concentrations of $\mathrm{MgCl}_{2}$, $\mathrm{CaCl}_{2}$, and BSA were tested. The presence of $\mathrm{Mg}$ or Ca ions enhanced CD30 aptamer binding to cultured Karpas 299 cells with a maximal effect at a final concentration of $1 \mathrm{mM} \mathrm{Mg}^{2+}$ or $\mathrm{Ca}^{2+}$ (Figures 2a and b), but had little effect on nonspecific aptamer binding to Jurkat cells. The addition of BSA resulted in a significant increase in CD30 aptamer binding to Karpas 299 cells with a maximal effect at $0.1 \%$ concentration, and also reduced nonspecific binding to Jurkat cells (Figure 2c), thereby increasing the overall specificity of CD30 aptamer-cell binding. Similar CD30 aptamer-cell binding patterns were observed with SUDHL-1 cells (data not shown). Thus, PBS buffer containing $1 \mathrm{mM} \mathrm{MgCl}_{2}$ and $0.1 \%$ BSA was used as the standard binding buffer in all further experiments. Thereafter, a dosing study was performed to determine optimal concentration of CD30 aptamer for cell binding. As shown in Figures 2d and 2e, CD30 aptamer had a high affinity to Karpas 299 and SUDHL-1 cells and reached saturation after $1 \mathrm{nM}$. In contrast, the CD30 aptamer showed minimal nonspecific binding to Jurkat cells (Figure 2f).

To rule out nonspecific binding of RNA aptamer to cultured cells, a 39-mer randomized control RNA aptamer probe was synthesized (Figure 1a) and tested. As expected, flow cytometry analysis showed that this randomized control probe had little 
to no binding capacity to Jurkat and Karpas 299 cells (Figures $3 a-d)$. To further validate cell-specific binding of the CD30 aptamer, fluorescent microscopic examination was carried out.

Table 1 Comparison of anti-CD30 antibody and CD30 aptamer binding to various cell types

\begin{tabular}{|c|c|c|}
\hline Tested cells & $\begin{array}{c}\text { Anti-CD30 } \\
\text { antibody }\end{array}$ & $\begin{array}{l}\text { CD30 } \\
\text { aptamer }\end{array}$ \\
\hline \multicolumn{3}{|l|}{ Cell lines } \\
\hline Jurkat (T-cell lymphoma) & - & - \\
\hline K562 (myeloid leukemia) & - & - \\
\hline RPMI8226 (multiple myeloma) & - & - \\
\hline SUDHL-1 (ALCL) & + & + \\
\hline Karpas 299 (ALCL) & + & + \\
\hline L-540 (Hodgkin lymphoma) & + & + \\
\hline HDLM2 (Hodgkin lymphoma) & + & + \\
\hline L-428 (Hodgkin lymphoma) & + & + \\
\hline KMH2 (Hodgkin lymphoma) & + & + \\
\hline \multicolumn{3}{|l|}{ Cells from lymphoma tissues* } \\
\hline Follicular lymphoma cells & - & - \\
\hline \multicolumn{3}{|l|}{ Cells from bone marrows** } \\
\hline Granulocytes & - & - \\
\hline Monocytes & - & - \\
\hline Lymphocytes & - & - \\
\hline Nuclear RBCs & - & - \\
\hline
\end{tabular}

${ }^{\star} n=5 ;{ }^{*} n=10$. RBCs, red blood cells.
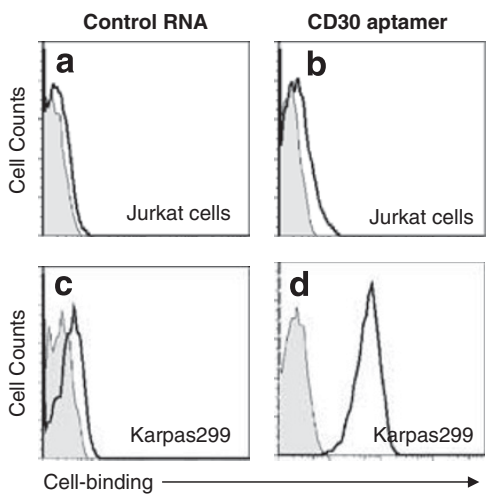

For this purpose, Karpas 299 cells were mixed with CD30negative Jurkat cells that had been pre-stained with CFSE. The cell mixtures were then incubated with the Cy5-labeled CD30 aptamer probe. Fluorescent microscopy (Figure 3e) showed that the CD30 aptamer probe specifically bound to Karpas 299 cells (the cells with unstained cytoplasm and red fluorescent signal on surface), but did not bind to the CFSE-labeled Jurkat cells (the cells with green fluorescent cytoplasm and no red fluorescent signal on the cell surface).

\section{Identical Cell Binding Specificity and Sensitivity by the CD30 Aptamer Probe and Anti-CD30 Antibody}

Currently, antibody staining is the standard histological method to detect CD30 expression in clinical tissues. To confirm specific cell binding, the aptamer was checked with a series of CD30-expressing cell lines, including human ALCL cells (SUDHL-1 and Karpas 299) and Hodgkin's lymphoma cells (L540, HDLM2, L428, and KMH2), and CD30-negative cell lines, including T-cell leukemia cells (Jurkat), myeloid leukemia cells (K562), and multiple myeloma cells (RPMI8226). Cells were incubated with the $0.3 \mathrm{nM}$ Cy5-labeled CD30 aptamer or FITC-labeled anti-CD30 antibody in binding buffer for $20 \mathrm{~min}$. Cell binding by the aptamer and the antibody was analyzed by flow cytometry. Both the CD30 aptamer probe and anti-CD30 antibody showed identical cell binding patterns. Strong staining was seen with all tested CD30-expressing cells (SUDHL-1, Karpas 299, L340, HDLM2, L428, and KMH2 cells), whereas little or no staining was seen with CD30-negative cells (Jurkat, K562, and RPMI8226 cells) (Figure 4; Table 1).

For further validation, nuclear cells, including granulocytes, monocytes, lymphocytes, and nuclear RBCs, from marrow aspirates of healthy donors $(n=10)$ were prepared. Cells from fresh lymph node tissue sections of follicular lymphoma patients $(n=5)$ were also prepared as described

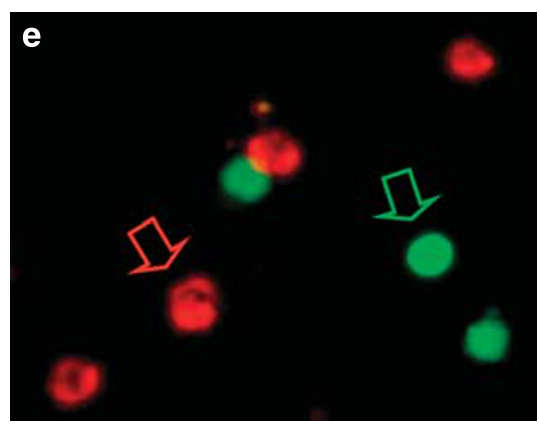

Figure 3 Specific cell staining by the CD30 aptamer. (a-d) Cell binding assays. To rule out nonspecific cell binding, a 39-mer control RNA probe with a random sequence was synthesized (Figure 1a) and incubated with cultured lymphoma cells. The resultant aptamer-cell binding was examined by flow cytometry. Gray peaks represent unstained cells, and open peaks represent cells that were stained with the aptamer probes as indicated. (e) Fluorescent cell staining by the CD30 aptamer. Jurkat cells were pre-labeled with the fluorescent dye CFSE and mixed with fresh, untreated Karpas 299 cells. Cell mixtures were then incubated with the Cy5-labeled CD30 aptamer, and the resultant aptamer-cell staining was examined under a fluorescent microscope. Pre-labeled Jurkat cells were identified by green fluorescence and are indicated by the green arrow, and Karpas 299 cells that were specifically stained by CD30 aptamer were identified by red fluorescence and are indicated by the red arrow. 

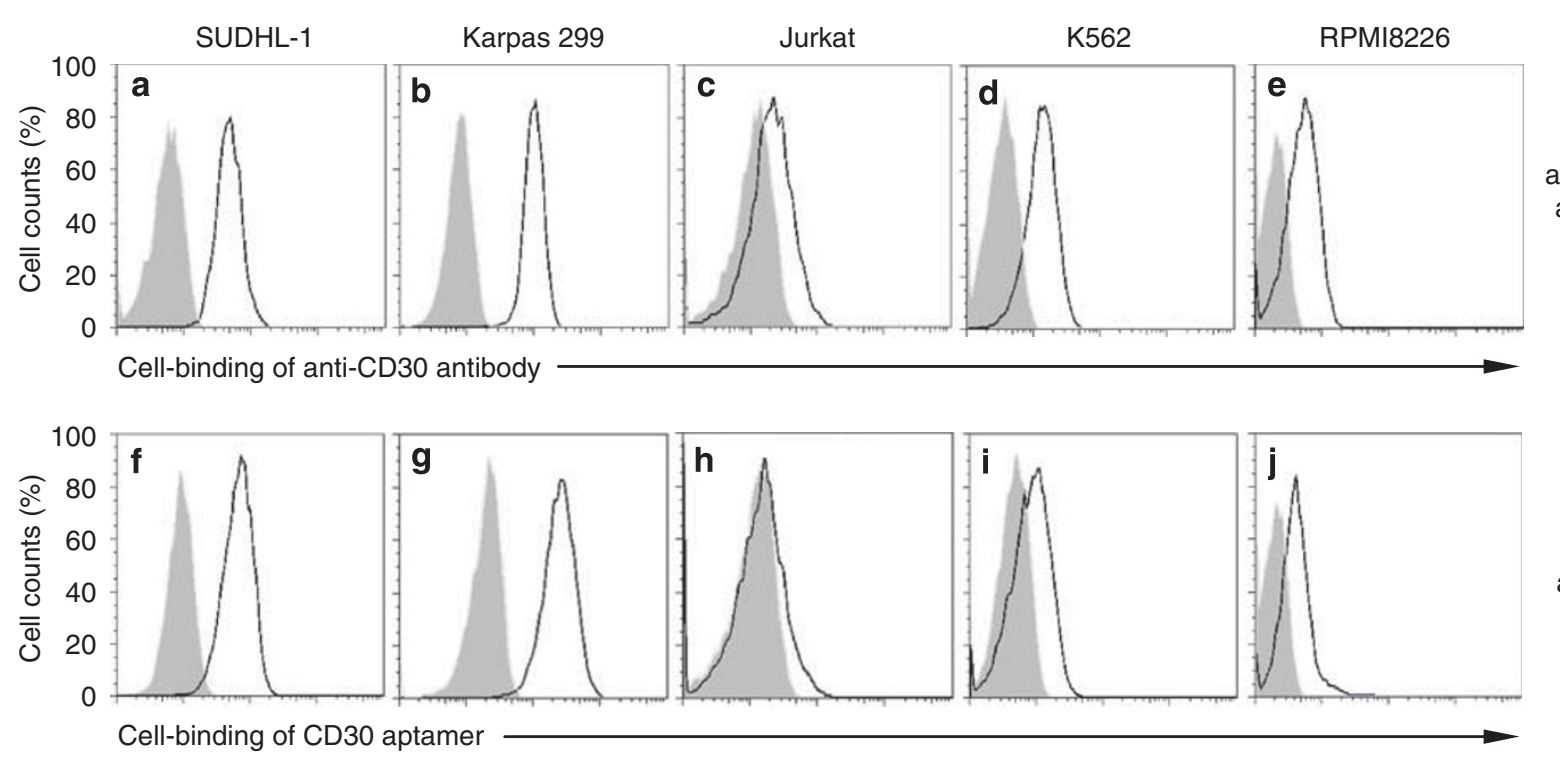

anti-CD30

antibody

CD30

aptamer

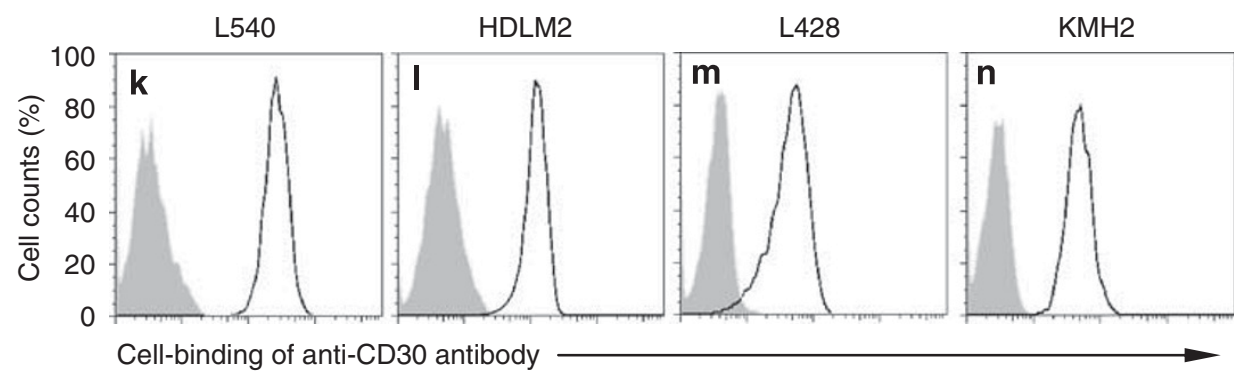

anti-CD30
antibody
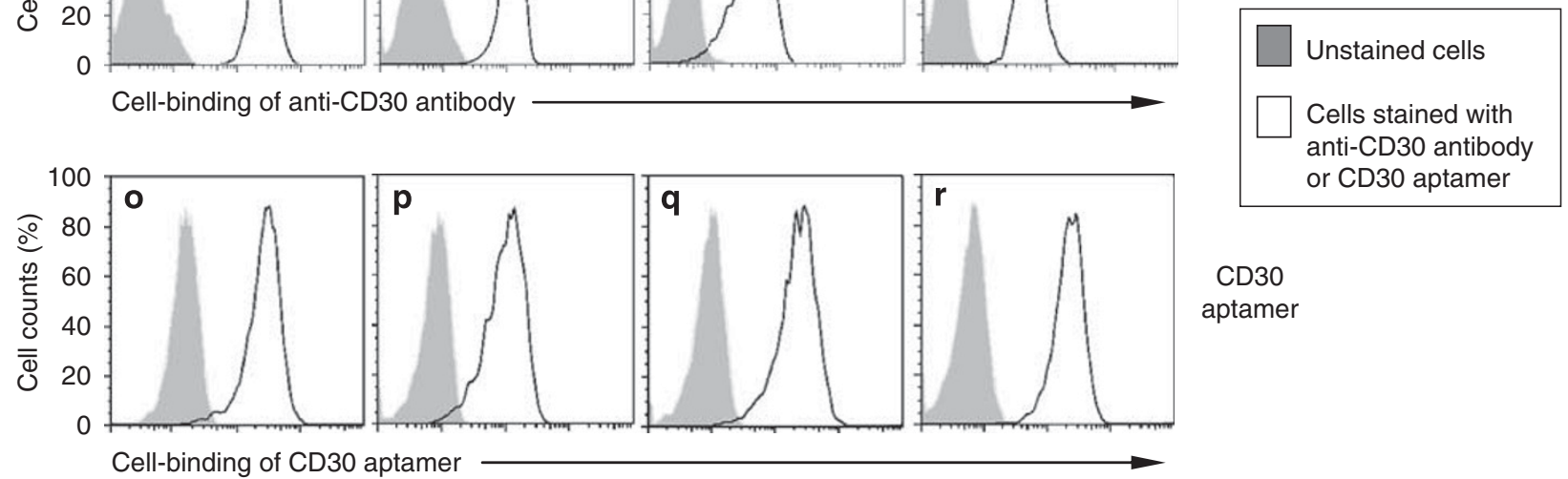

CD30

aptamer

Figure 4 Identical cell binding patterns of the CD30 aptamer and anti-CD30 antibody. Cultured human lymphoma and leukemia cells (as indicated) were incubated in binding buffer containing the FITC-labeled anti-CD30 antibody (a-e and $\mathbf{k}-\mathbf{n}$ ) or Cy5-labeled CD30 aptamer (f-j and $\mathbf{o}-\mathbf{r}$ ) for 20 min. Cell binding was examined by flow cytometry analysis. Gray peaks represent unstained cells, and open peaks represent cells that were stained by the aptamer or antibody.

in 'Materials and Methods.' The fresh cells were probed by the AmCyan-labeled anti-CD45 (common leukocyte antigen) antibody and Cy5-labeled CD30 aptamer. In the control group, the FITC-labeled anti-CD30 antibody was used instead of the CD30 aptamer. Stained cells were analyzed by flow cytometry, and populations of granulocytes, monocytes, and lymphocytes were gated according to their distribution on the side scatter vs CD45 panels. Subsequently, cell binding of the CD30 aptamer to the gated individual cell populations was evaluated and compared with that of the anti-CD30 antibody. The CD30 targeting aptamer and the anti-CD30 antibody showed identical cell staining patterns (Table 1).

\section{Competition Between the CD30-Specific Aptamer and Anti-CD30 Antibody}

To examine whether the CD30 aptamer probe and anti-CD30 antibody competitively bind to the CD30 protein, cultured Karpas 299 cells were simultaneously incubated with the Cy5labeled CD30 aptamer probe and FITC-labeled anti-CD30 antibody at room temperature for $20 \mathrm{~min}$. As shown in Figures $5 \mathrm{a}-\mathrm{d}$, both the CD30 aptamer and anti-CD30 antibody independently bound to the $\mathrm{CD} 30$ proteins on the surface of Karpas 299 cells, suggesting that these two probes recognize different epitopes/regions of CD30 molecules, although the negative control Jurkat cells that do 

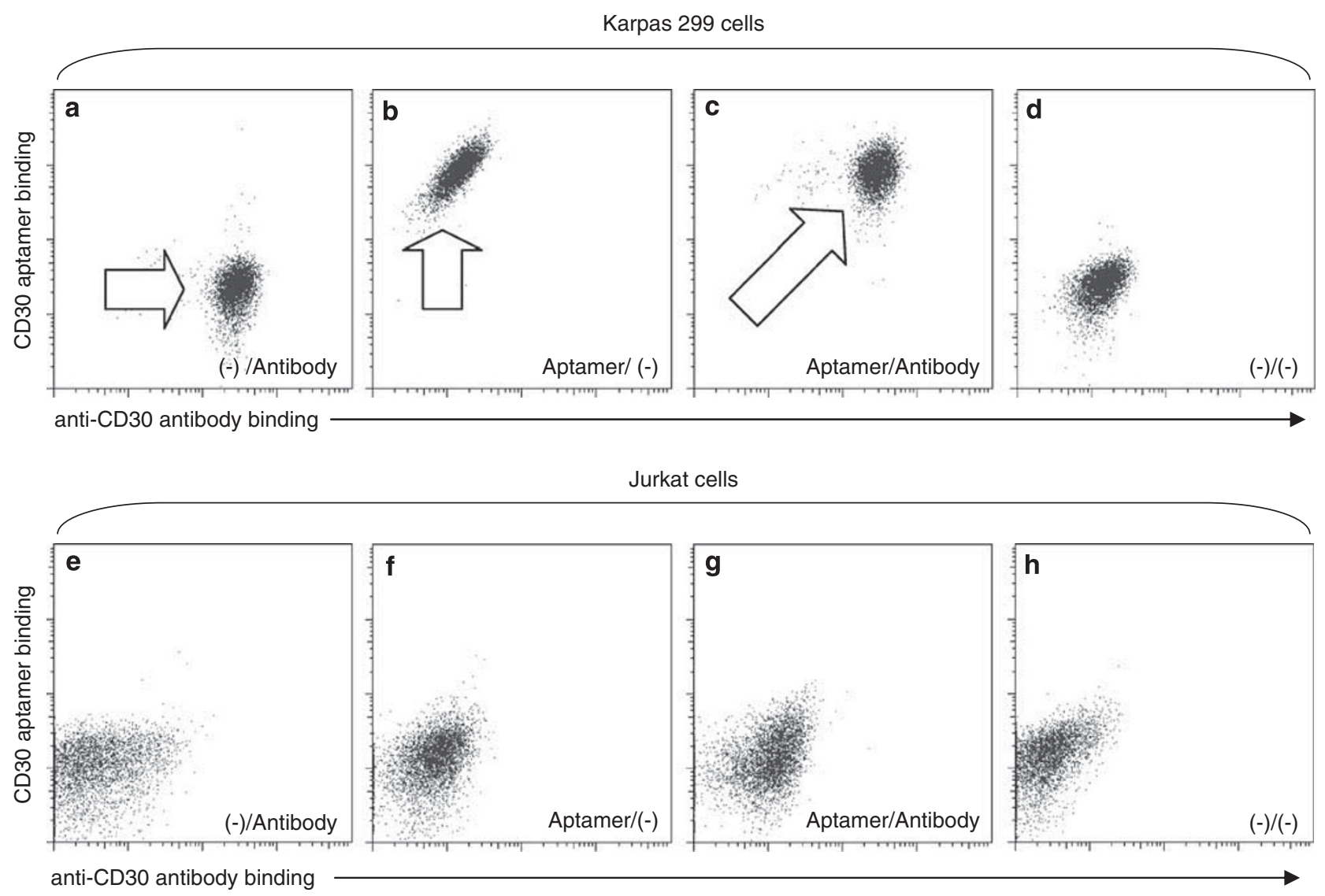

Figure 5 Double staining of cells by the CD30 aptamer and anti-CD30 antibody. Cultured Karpas 299 cells (a-d) and Jurkat cells (e-h) were incubated with the Cy5-labeled CD30 aptamer, FITC-labeled anti-CD30 antibody, or both simultaneously. Resultant cell staining was examined by flow cytometry analysis. Stained cell populations are indicated by arrows.

not express CD30 showed no label with either probes (Figures 5e-h).

\section{Immunophenotyping of Cells by a Combination of the CD30 Aptamer and Antibodies}

Advanced flow cytometry enables simultaneous detection of multiple biomarkers on individual cells. Specific type of cells could be identified and isolated by simultaneously using multiple specific probes that are labeled with different fluorochromes. Thus, it is interesting to determine whether the CD30 aptamer probe could be used in combination with other antibodies for multicolor cell staining. To answer this question, cells were incubated with a staining solution consisting of the Cy5-labeled aptamer probe specific for CD30 (a cell marker for ALCL and Hodgkin's lymphoma), and with a PE-labeled antibody specific for CD25 (a lymphocyte activation marker that is expressed by activated $\mathrm{T}$ and $\mathrm{B}$ cells as well as ALCL cells), AmCyan-labeled antibody specific for CD45, or FITC-labeled antibody specific for CD30. As shown in Figures 6a-f, Karpas 299 cells and SUDHL-1 cells were simultaneously stained by the CD30 aptamer probe and antibodies against CD25, CD30, and CD45. In the control group, Jurkat cells showed no staining by antibodies to CD25 or CD30 (both aptamer and antibody probes), but positive staining by the CD45 antibody as expected (Figures $6 \mathrm{~g}-\mathrm{i}$ ).

\section{Detection of ALCL Cells by the CD30 Aptamer Probe in a Mixed Cell Specimen}

To validate whether the $\mathrm{CD} 30$ aptamer probe could be used in detecting ALCL cells from a mixed background of cells, a process more typical of the flow cytometry immunophenotyping for clinical lymphoma diagnosis, we used fresh bone marrow nucleated cells from healthy donors spiked with CD30-positive ALCL cells (Karpas 299). The samples were prepared and stained simultaneously with the Cy5-labeled CD30 aptamer, AmCyan-labeled anti-CD45 antibody, and FITC-labeled anti-CD30 antibody as described in 'Materials and Methods.' The populations of nucleated RBCs, blasts, lymphocytes, granulocytes, monocytes, and Karpas 299 cells were separated and gated according to their distribution on the side scatter (SSC) vs CD45 panels (Figure 7a). Cellular CD30 expression in each gated cell population was then evaluated by the CD30 aptamer or antiCD30 antibody vs CD45 panels. As illustrated in Figures $7 \mathrm{~b}$ and $c$, the CD30 aptamer and anti-CD30 antibody showed nearly identical staining patterns and numbers for CD30- 

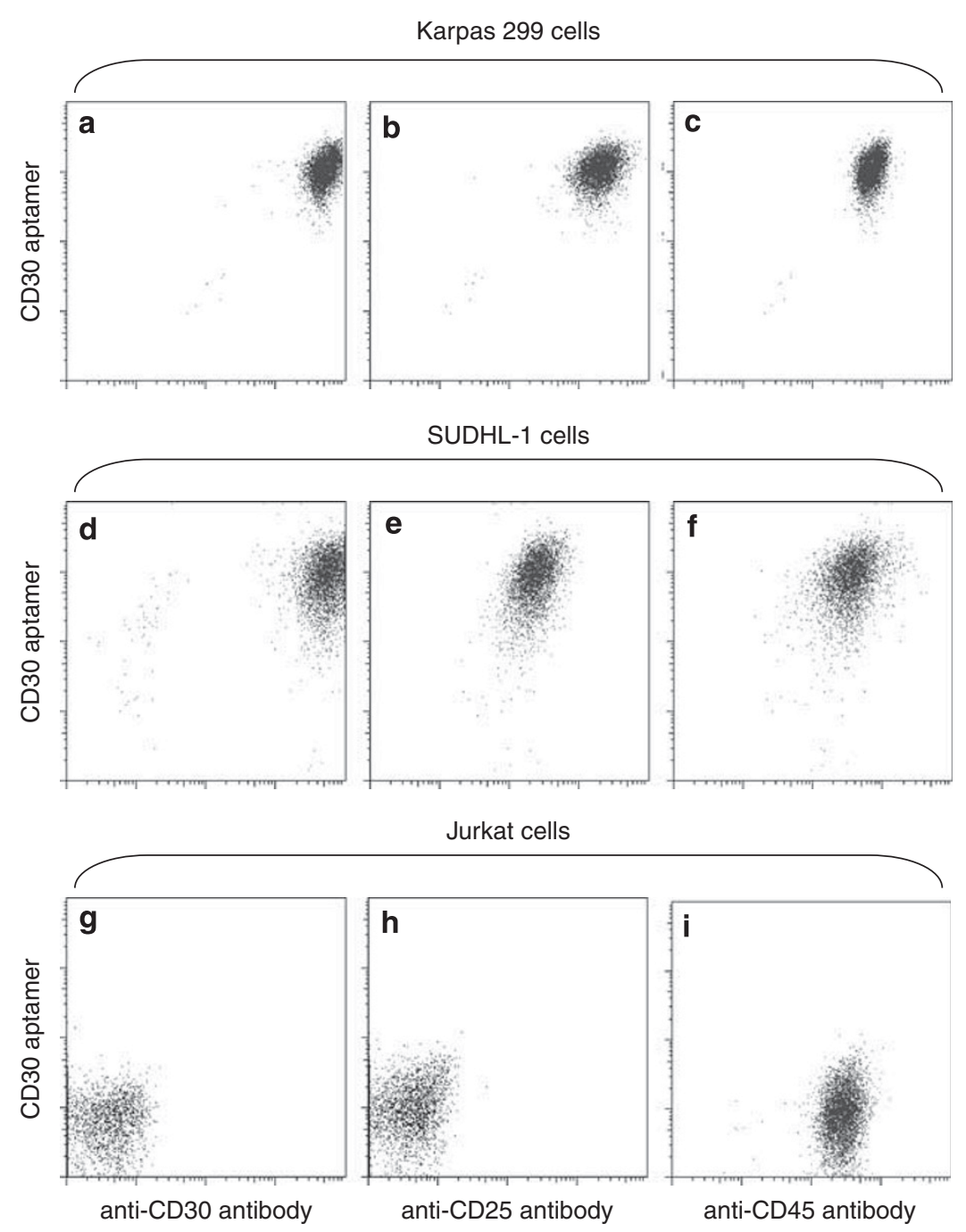

Figure 6 Multicolor staining of cells by combined exposure to the CD30 aptamer and antibodies. Cultured Karpas 299 cells (a-c), SUDHL-1 cells (d-f), and Jurkat cells (g-i) were incubated with mixed probes, including the Cy5-labeled CD30 aptamer, PE-labeled anti-CD25 antibody, FITC-labeled anti-CD30 antibody, and AmCyan-labeled anti-CD45 antibody. Resultant cell staining was examined by multicolor flow cytometry analysis.

positive Karpas 299 cells as well as CD30-negative background cells.

\section{DISCUSSION}

Currently, antibodies are generally the only available clinical probes for lymphoma diagnosis. ${ }^{25}$ The study has shown that an RNA aptamer possesses identical specificity and sensitivity to those of anti-CD30 antibodies. Our results suggested that the CD30-specific aptamer could replace the anti-CD30 antibody in flow cytometry analysis. In addition, multicolor staining of cultured cells indicates that the CD30 aptamer probe can be used in combination with antibodies in multiparameter flow cytometry analysis for lymphoma diagnosis. It will be interesting to explore the use of CD30 aptamer probes for immunohistochemical staining of fixed tissue sections for the diagnoses of $\mathrm{CD} 30$-associated diseases, including ALCL and Hodgkin's Lymphomas. Before clinical application of the CD30 aptamer probe, a large-scale validation study with samples from both normal donors and lymphoma patients should be performed.

In comparison with antibodies, oligonucleotide aptamers, such as those synthesized in this work, have unparalleled advantages for clinical use. As a small molecule, aptamers are not antigenic and can be synthesized at a significantly lower cost than antibodies. ${ }^{26}$ In addition, aptamers can be easily modified and labeled with fluorescent, optical, or radioisotope reporters as needed for tracking purposes. ${ }^{4,6,7,23}$ These features increase the potential of aptamers for the in vivo application as diagnostic/therapeutic agents. Our findings indicate that the aptamer probe can be used in combination with antibodies for multicolor cell immunophenotyping analysis, and it may be a suitable replacement for the anti-CD30 antibody in disease diagnosis (Figure 7). In addition, studies using antibodies have revealed that the binding of the $\mathrm{CD} 30$ receptors results in the activation of the cellular signaling pathways that lead to the growth arrest and 

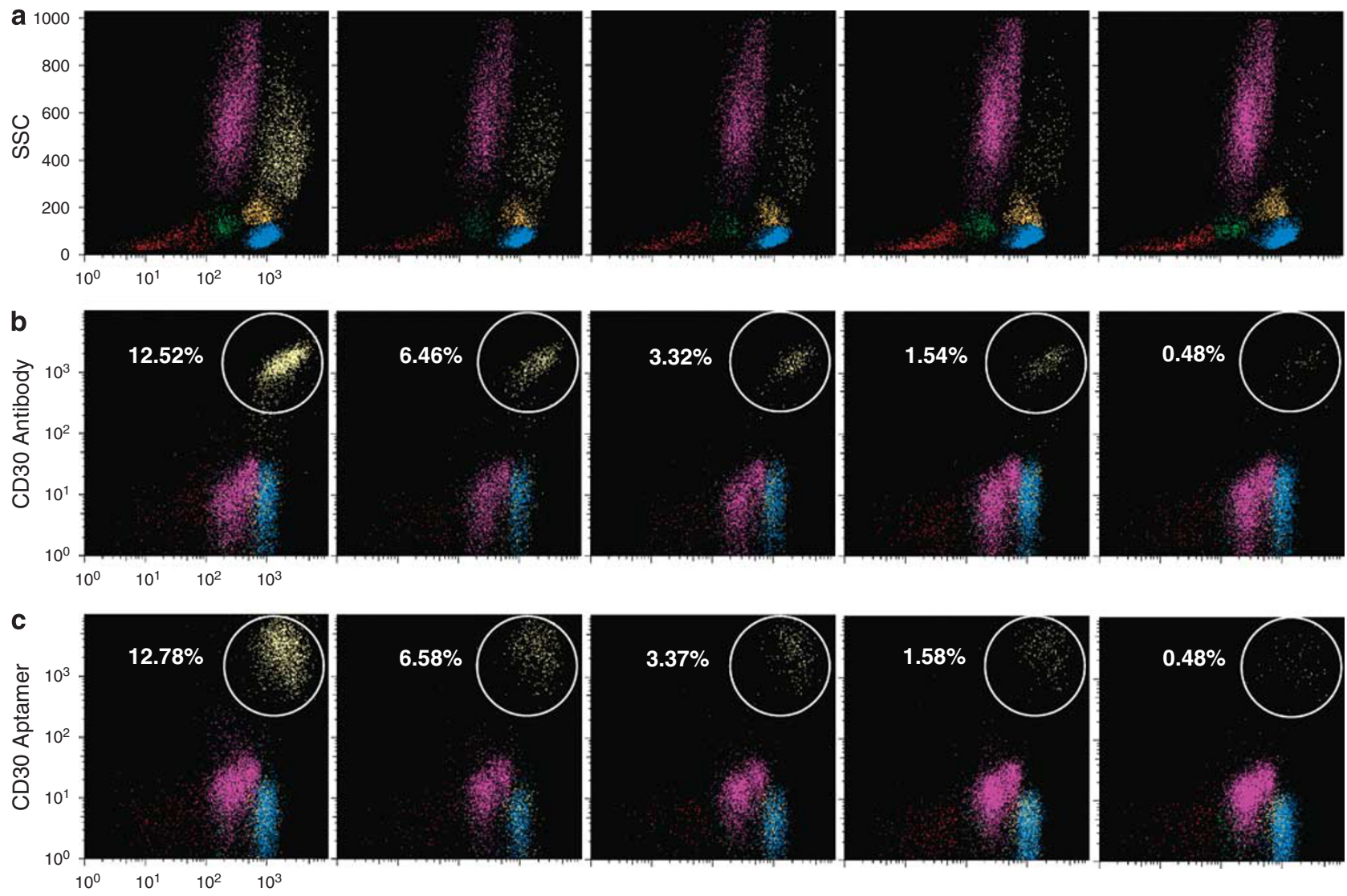

\section{CD45}

Nucleated red blood cells

Blasts

Lymphocytes

Granulocytes

Monocytes

Karpas 299 cells

Figure 7 Detection of ALCL cells by the CD30 aptamer probe in a mixed cell specimen. (a) A mixture of fresh bone marrow nucleated cells and cultured Karpas 299 cells was stained simultaneously with the CD30 aptamer probe, anti-CD45 antibody, and anti-CD30 antibody. The populations of nucleated RBCs, blasts, lymphocytes, granulocytes, monocytes, and admixed Karpas 299 cells were separated and gated (painted in a different color as listed on the bottom) according to their distribution on the side scatter (SSC) vs CD45 panels. (b) CD30 expressions of individual cell populations gated in (a) were then examined by the anti-CD30 antibody vs CD45 panels and the numbers of detected CD30-positive Karpas 299 cells were indicated in each sample. (c) CD30 aptamer probe vs CD45 panels and the numbers of detected CD30-positive Karpas 299 cells.

death of ALCL cells in vitro. ${ }^{20,27-29}$ These findings suggest that the CD30 receptors may also be a therapeutic target for ALCL treatment. To understand whether the binding of aptamers to the CD30 receptors has any intracellular effect, we have exposed cultured ALCL cells (Karpas 299 and SUDHL-1) to the CD30 aptamer; however, the synthesized aptamer had no effect on cell growth as assessed by the MTT assay (unpublished result). It is notable that in contrast to antibodies that contain two binding sites to the $\mathrm{CD} 30$ receptors, the $\mathrm{CD} 30$ aptamer probes have only a single binding site that is not able to crosslink and activate the CD30 receptors on the cell surface.

Aptamers, especially the RNA-based aptamers, are susceptible to degradation by environmental nucleases, largely limiting their clinical value to date. However, chemical modification with 2'-O-methyl or 2-fluoro nucleic acids, phosphorothioate linkages, or a combination of these has been reported to significantly improve the nuclease resistance of the RNA-based aptamers. ${ }^{30-34}$ Considering the rapidly advancing synthesis technologies, we believe that aptamers could become powerful diagnostic and therapeutic agents in the near future.

\section{ACKNOWLEDGEMENTS}

This work was supported by the National Institutes of Health (grant 5K22CA113493 to YZ), BCM-Methodist lymphoma SPORE (developing projects awarded to $\mathrm{YZ}$ and $\mathrm{CHT}$ ), and TMHRI scholar and Clinical Translation Study Award (to YZ). Funding for open access charge: the NIH grant 5K22CA113493.

\section{DISCLOSURE/CONFLICT OF INTEREST}

The authors declare no conflict of interest.

1. Ulrich $\mathrm{H}$, Martins $\mathrm{AH}$, Pesquero JB. RNA and DNA aptamers in cytomics analysis. Cytometry A 2004;59:220-231.

2. Ellington AD, Szostak JW. In vitro selection of RNA molecules that bind specific ligands. Nature 1990;346:818-822.

3. Tuerk C, Gold L. Systematic evolution of ligands by exponential enrichment: RNA ligands to bacteriophage T4 DNA polymerase. Science (NY) 1990;249:505-510.

4. Brody EN, Gold L. Aptamers as therapeutic and diagnostic agents. J Biotechnol 2000;74:5-13. 
5. Fredriksson S, Gullberg $M$, Jarvius J, et al. Protein detection using proximity-dependent DNA ligation assays. Nat Biotechnol 2002;20:473-477.

6. Nimjee SM, Rusconi CP, Sullenger BA. Aptamers: an emerging class of therapeutics. Annu Rev Med 2005;56:555-583.

7. Bunka DH, Stockley PG. Aptamers come of age-at last. Nat Rev 2006;4:588-596.

8. Cerchia L, Hamm J, Libri D, et al. Nucleic acid aptamers in cancer medicine. FEBS Lett 2002;528:12-16.

9. Mori T, Oguro A, Ohtsu T, et al. RNA aptamers selected against the receptor activator of NF-kappaB acquire general affinity to proteins of the tumor necrosis factor receptor family. Nucleic Acids Res 2004;32:6120-6128.

10. Nawrocki JF, Kirsten ES, Fisher RI. Biochemical and structural properties of a Hodgkin's disease-related membrane protein. J Immunol 1988;141:672-680.

11. Stein H, Mason DY, Gerdes J, et al. The expression of the Hodgkin's disease associated antigen $\mathrm{Ki}-1$ in reactive and neoplastic lymphoid tissue: evidence that Reed-Sternberg cells and histiocytic malignancies are derived from activated lymphoid cells. Blood 1985;66:848-858.

12. Chiarle R, Podda A, Prolla G, et al. CD30 in normal and neoplastic cells. Clin Immunol 1999;90:157-164.

13. Delsol GJE, Faini B, Gascoyne RD, et al. Anaplastic large cell lymphoma (ALCL), ALK positive. In: Swerdlow SH CE, Harris NL, Jaffe ES, Pileri SA, Stein $\mathrm{H}$, Thiele J, Vardiman JW (eds). WHO Classification of Tumors of Haematopoietic and Lymphod Tissues. WHO Press: Lyon, France, 2008, pp 312-316.

14. Medeiros LJ, Elenitoba-Johnson KS. Anaplastic large cell lymphoma. Am J Clin Pathol 2007;127:707-722.

15. Staber PB, Noehammer C, Durkop $\mathrm{H}$, et al. mRNA expression patterns indicate CD30 mediated activation of different apoptosis pathways in anaplastic large cell lymphoma but not in Hodgkin's lymphoma. Leuk Res 2006;30:343-348.

16. Hirsch B, Hummel M, Bentink S, et al. CD30-induced signaling is absent in Hodgkin's cells but present in anaplastic large cell lymphoma cells. Am J Pathol 2008;172:510-520.

17. Wendtner CM, Schmitt B, Gruss HJ, et al. CD30 ligand signal transduction involves activation of a tyrosine kinase and of mitogenactivated protein kinase in a Hodgkin's lymphoma cell line. Cancer Res 1995;55:4157-4161.

18. Hubinger G, Muller $\mathrm{E}$, Scheffrahn I, et al. CD30-mediated cell cycle arrest associated with induced expression of p21(CIP1/WAF1) in the anaplastic large cell lymphoma cell line Karpas 299. Oncogene 2001;20:590-598.

19. Levi E, Pfeifer WM, Kadin ME. CD30-activation-mediated growth inhibition of anaplastic large-cell lymphoma cell lines: apoptosis or cell-cycle arrest? Blood 2001;98:1630-1632.
20. Mir SS, Richter BW, Duckett CS. Differential effects of CD30 activation in anaplastic large cell lymphoma and Hodgkin disease cells. Blood 2000;96:4307-4312.

21. Nishikori $M$, Ohno $H$, Haga $H$, et al. Stimulation of CD30 in anaplastic large cell lymphoma leads to production of nuclear factor-kappaB p52, which is associated with hyperphosphorylated Bcl-3. Cancer Sci 2005;96:487-497.

22. Hubinger G, Schneider C, Stohr D, et al. CD30-induced up-regulation of the inhibitor of apoptosis genes CIAP1 and CIAP2 in anaplastic large cell lymphoma cells. Exp Hematol 2004;32:382-389.

23. Mukhopadhyay R. APTAMERS ARE READY FOR. Anal Chem 2005;77:114A-118A

24. Hodgkin PD, Lee JH, Lyons AB. B cell differentiation and isotype switching is related to division cycle number. J Exp Med 1996;184: 277-281.

25. Stewart CC, Behm FG, Carey JL, et al. US-Canadian consensus recommendations on the immunophenotypic analysis of hematologic neoplasia by flow cytometry: selection of antibody combinations. Cytometry 1997;30:231-235.

26. Kaur G, Roy I. Therapeutic applications of aptamers. Expert Opin Investig Drugs 2008;17:43-60.

27. Schneider C, Hubinger G. Pleiotropic signal transduction mediated by human CD30: a member of the tumor necrosis factor receptor (TNFR) family. Leuk Lymphoma 2002;43:1355-1366.

28. Heuck F, Ellermann J, Borchmann $\mathrm{P}$, et al. Combination of the human anti-CD30 antibody 5F11 with cytostatic drugs enhances its antitumor activity against Hodgkin and anaplastic large cell lymphoma cell lines. J Immunother 2004;27:347-353.

29. Wahl AF, Klussman K, Thompson JD, et al. The anti-CD30 monoclonal antibody SGN-30 promotes growth arrest and DNA fragmentation in vitro and affects antitumor activity in models of Hodgkin's disease. Cancer Res 2002;62:3736-3742.

30. Kusser W. Chemically modified nucleic acid aptamers for in vitro selections: evolving evolution. J Biotechnol 2000;74: 27-38.

31. Ulrich H. RNA aptamers: from basic science towards therapy. Handb Exp Pharmacol 2006;305-326.

32. Stoltenburg R, Reinemann C, Strehlitz B. SELEX-a (r)evolutionary method to generate high-affinity nucleic acid ligands. Biomol Eng 2007:24:381-403.

33. Keefe AD, Cload ST. SELEX with modified nucleotides. Curr Opin Chem Biol 2008;12:448-456.

34. Minakawa N, Sanji M, Kato $Y$, et al. Investigations toward the selection of fully-modified 4'-thioRNA aptamers: optimization of in vitro transcription steps in the presence of $4^{\prime}$-thioNTPs. Bioorg Med Chem 2008;16:9450-9456. 